Michael Dickman, DO, FAAFP; Bob Marshall, MD, MPH, MISM, FAAFP, FAMIA; Erica $S$. Meisenheimer, MD Madigan Family Medicine Residency, Joint Base Lewis-McChord, WA

DEPUTY EDITOR Jennie B. Jarrett, PharmD, BCPS, MMedEd, FCCP University of Illinois at Chicago

\section{Is it better to take that antihypertensive at night?}

\section{PRACTICE CHANGER}

Advise patients to take blood pressure (BP) medication at bedtime rather than upon waking because it results in a decrease in major cardiovascular disease events.

STRENGTH OF RECOMMENDATION

B: Based on a single, good-quality, multicenter trial.

Hermida RC, Crespo JJ, Domínguez-Sardiña M, et al. Bedtime hypertension treatment improves cardiovascular risk reduction: the Hygia Chronotherapy Trial [published online ahead of print October 22, 2019]. Eur Heart J. 2019; ehz754. doi:10.1093/eurheartj/ehz754. ${ }^{1}$

\section{ILLUSTRATIVE CASE}

A 54-year-old White woman presents to your office with new-onset hypertension. As you are discussing options for treatment, she mentions she would prefer once-daily dosing to help her remember to take her medication. She also wants to know what the best time of day is to take her medication to reduce her risk of cardiovascular disease (CVD). What do you advise?

$\mathrm{T}$ he burden of hypertension is significant and growing in the United States. The 2017 American College of Cardiology/American Heart Association (ACC/AHA) guidelines reported that more than 108 million people were affected in 2015-2016-up from 87 million in 1999-2000. ${ }^{2}$ Yet control of hypertension is improving among those receiving antihypertension pharmacotherapy. As reported in the ACC/AHA guidelines, data from the 2016 National Health and $\mathrm{Nu}$ trition Examination Survey (NHANES) indicate an increase of controlled hypertension among those receiving treatment from $25.6 \%$ (1999-2000) to $43.5 \%(2015-2016){ }^{2}$

Chronotherapy involves the administration of medication in coordination with the body's circadian rhythms to maximize therapeutic effectiveness and/or minimize adverse effects. It is not a new concept as it applies to hypertension. Circadian rhythmdependent mechanisms influence the natural rise and fall of blood pressure (BP). ${ }^{1}$ The renin-angiotensin-aldosterone system, known to be most active at night, is a target mechanism for BP control. ${ }^{1}$ Angiotensin-converting enzyme (ACE) inhibitors and angiotensin II receptor blockers (ARBs) are more effective (alone or in combination with other agents) at reducing BP during sleep and wakefulness when they are taken at night. ${ }^{3,4}$ Additional prospective clinical trials and systematic reviews have documented improved BP during sleep and on 24-hour ambulatory monitoring when antihypertensives are taken at bedtime..$^{3-5}$

However, there have been few long-term studies assessing the effects of bedtime administration of antihypertensive medication on CVD risk reduction with patient-oriented outcomes. $^{6,7}$ Additionally, no studies have evaluated morning vs bedtime administration of antihypertensive medication for CVD risk reduction in a primary care setting. The 2019 ACC/AHA guideline on the primary prevention of CVD offers no recommenda- 
tion regarding when to take antihypertensive medication. ${ }^{8}$ Timing of medication administration also is not addressed in the NHANES study of hypertension awareness, treatment, and control in US adults. ${ }^{9}$

This study sought to determine in a primary care setting whether taking antihypertensives at bedtime, as opposed to upon waking, more effectively reduces CVD risk.

\section{STUDY SUMMARY}

\section{PM vs AM antihypertensive dosing reduces $\mathrm{CV}$ events}

This prospective, randomized, open-label, blinded endpoint trial of antihypertensive medication administration timing was part of a large, multicenter Spanish study investigating ambulatory $\mathrm{BP}$ monitoring (ABPM) as a routine diagnostic tool.

Study participants were randomly assigned in a 1:1 ratio to 2 treatment arms; participants either took all of their BP medications in the morning upon waking ( $n=9532)$ or right before bedtime $(n=9552)$. The study was conducted in a primary care clinical setting. It included adult participants (age $\geq 18$ years) with hypertension (defined as having at least 1 of the following benchmarks: awake systolic BP [SBP] mean $\geq 135 \mathrm{~mm} \mathrm{Hg}$, awake diastolic BP (DBP) mean $\geq 85 \mathrm{~mm} \mathrm{Hg}$, asleep SBP mean $\geq 120 \mathrm{~mm} \mathrm{Hg}$, asleep DBP mean $\geq 70 \mathrm{~mm} \mathrm{Hg}$ as corroborated by 48 -hour ABPM) who were taking at least 1 antihypertensive medication.

Any antihypertension medication included in the Spanish national formulary was allowed (exact agents were not delineated, but the following classes were included: $\mathrm{ARB}, \mathrm{ACE}$ inhibitor, calcium channel blocker [CCB], beta-blocker, and/or diuretic). All BP medications had to be dosed once daily for inclusion. Exclusion criteria included pregnancy, night or rotating-shift work, alcohol or other substance dependence, acquired immunodeficiency syndrome, preexisting CVD (unstable angina, heart failure, arrhythmia, kidney failure, and retinopathy), inability to tolerate ABPM, and inability to comply with required 1-year follow-up.

Upon enrollment and at every subsequent clinic visit (scheduled at least annually), participants underwent 48-hour ABPM. Those with uncontrolled BP or elevated CVD risk had scheduled follow-up and ABPM more frequently. The primary outcome was a composite of CVD events including new-onset myocardial infarction, coronary revascularization, heart failure, ischemic stroke, hemorrhagic stroke, and CVD death. Secondary endpoints were individually analyzed primary outcomes of CVD events. The typical patient at baseline was 60.5 years of age with a body mass index of 29.7, an almost 9-year duration of hypertension, and a baseline office BP of 149/86 mm Hg. The patient break-out by antihypertensive class (awakening vs bedtime groups) was as follows: ARB (53\% vs 53\%), ACE inhibitor (25\% vs $23 \%$ ), CCB (33\% vs $37 \%$ ), beta-blocker ( $22 \%$ vs $18 \%$ ), and diuretic ( $47 \%$ vs $40 \%$ ).

During the median 6.3-year patient follow-up period, 1752 participants experienced a total of 2454 CVD events. Patients in the bedtime administration group, compared with those in the morning group, showed significantly lower risk for a CVD event (hazard ratio $[\mathrm{HR}]=0.55 ; 95 \%$ confidence interval $[\mathrm{CI}]$, $0.50-0.61 ; P<.001)$. Also, there was a lower risk for individual CVD events in the bedtime administration group: CVD death $(\mathrm{HR}=0.44$; 95\% CI, 0.34-0.56), myocardial infarction $(\mathrm{HR}=0.66$; 95\% CI, 0.52-0.84), coronary revascularization $(\mathrm{HR}=0.60 ; 95 \% \mathrm{CI}, 0.47-0.75)$, heart failure ( $\mathrm{HR}=0.58 ; 95 \% \mathrm{CI}, 0.49-0.70)$, and stroke (HR $=0.51 ; 95 \% \mathrm{CI}, 0.41-0.63)$. This difference remained after correction for multiple potential confounders. There were no differences in adverse events, such as sleeptime hypotension, between groups.

\section{WHAT'S NEW}

\section{First RCT in primary care to show} dosing time change reduces $\mathrm{CV}$ risk

This is the first randomized controlled trial (RCT) performed in a primary care setting to compare before-bedtime to upon-waking administration of antihypertensive medications using clinically significant endpoints. The study demonstrates that a simple change in administration time has the potential to significantly improve the lives of our patients by reducing the risk for cardiovascular events and their medication burden.
A simple change in administration time has the potential to significantly improve the lives of our patients by reducing the risk for cardiovascular events and their medication burden. 


\section{CAVEATS}

\section{Homogenous population and} exclusions limit generalizability

Because the study population consisted of white Spanish men and women, the results may not be generalizable beyond that ethnic group. In addition, the study exclusions limit interpretation in night/rotating-shift employees, patients with secondary hypertension, and those with CVD, chronic kidney disease, or severe retinopathy looking to reduce their risk.

\section{CHALLENGES TO IMPLEMENTATION}

\section{Nighttime urination} could lead to nonadherence

Taking diuretics at bedtime may result in unwanted nighttime awakenings for visits to the bathroom, which could lead to nonadherence in some patients.

See "It"s time to change when BP meds are taken" on page 324 for more on the controversy that surrounded the initial release of this study.

\section{ACKNOWLEDGMENT}

The PURLs Surveillance System was supported in part by Grant Number UL1RR024999 from the National Center for Research Resources, a Clinical Translational Science Award to the University of Chicago. The content is solely the responsibility of the authors and does not necessarily represent the official views of the National Center for Research Resources or the National Institutes of Health. Copyright @ 2020. The Family Physicians Inquiries Network. All rights reserved.

\section{References}

1. Hermida RC, Crespo JJ, Domínguez-Sardiña M, et al. Bedtime hypertension treatment improves cardiovascular risk reduction: the Hygia Chronotherapy Trial [published online ahead of print October 22, 2019]. Eur Heart J. 2019;ehz754. doi:10.1093/ eurheartj/ehz754.

2. Dorans KS, Mills KT, Liu Y, et al. Trends in prevalence and control of hypertension according to the 2017 American College of Cardiology/American Heart Association (ACC/AHA) guideline. J Am Heart Assoc. 2018;7:e008888.

3. Hermida RC, Ayala DE, Smolensky MH, et al. Chronotherapy with conventional blood pressure medications improves management of hypertension and reduces cardiovascular and stroke risks. $\mathrm{Hy}$ pertens Res. 2016;39:277-292.

4. Bowles NP, Thosar SS, Herzig MX, et al. Chronotherapy for hypertension. Curr Hypertens Rep. 2018;20:97.

5. Zhao P, Xu P, Wan C, et al. Evening versus morning dosing regimen drug therapy for hypertension. Cochrane Database Syst Rev. 2011:CD004184.

6. Yusuf S, Sleight P, Pogue J, et al. Effects of an angiotensin-converting-enzyme inhibitor, ramipril, on cardiovascular events in highrisk patients: the Heart Outcomes Prevention Evaluation Study Investigators. NEngl J Med. 2000;342:145-153.

7. Black HR, Elliott WJ, Grandits G, et al. Principal results of the Controlled Onset Verapamil Investigation of Cardiovascular End Points (CONVINCE) trial. JAMA. 2003;289:2073-2082.

8. Arnette DK, Blumenthal RS, Albert MA, et al. 2019 ACC/AHA guideline on the primary prevention of cardiovascular disease: a report of the American College of Cardiology/American Heart Association Task Force on Clinical Practice Guidelines. J Am Coll Cardiol. 2019;74:e177-e232.

9. Foti K, Wang D, Appel LJ, et al. Hypertension awareness, treatment, and control in US adults: trends in the hypertensive control cascade by population subgroup (National Health and Nutrition Examination Survey, 1999-2016). Am J Epidemiol. 2019;188:2165-2174.
\Visit us @

mdedge.com/

familymedicine

(1)) Coronavirus vaccine: The contenders, the potential controversy

Doug Campos-Outcalt, MD, MPA

\section{(1)) Tackling unhealthy substance use using USPSTF guidance and a 1-question tool}

Doug Campos-Outcalt, MD, MPA

\section{ONLINE EXCLUSIVES}

- Your role in early diagnosis \& Tx of metastatic bone disease

- CASE REPORT 4-year-old girl • limited movement \& diffuse pain in both arms $\bullet$ pronated hands $\bullet$ Dx?

- CLINICAL INQUIRIES Which medications work best for menorrhagia?

- CLINICAL INQUIRIES Does evidence support the use of supplements to aid in BP control?

- RESIDENTS' RAPID REVIEW A 5-question monthly quiz to help you prepare for the family medicine certification exam. This month: EKGs

\section{PHOTO ROUNDS FRIDAY}

Test your diagnostic skills at www. mdedge.com/familymedicine/ photo-rounds

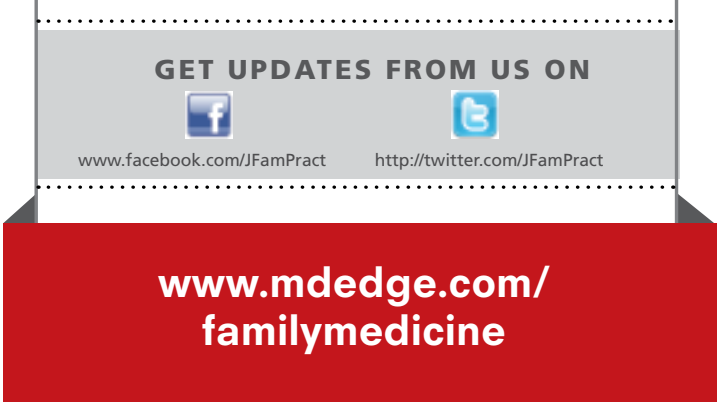

